\title{
Analyzing the Whole Transcriptome Profiles of ncRNAs and Predicting the Competing Endogenous RNA Networks in Cervical Cancer Cell Lines With Cisplatin Resistance
}

\section{Huimin Lv}

Third Hospital of Shanxi Medical University

\section{Shanshan Jin}

Third Hospital of Shanxi Medical University

\section{Binbin Zou}

Shanxi Medical University

Yuxiang Liang

Shanxi Medical University

Jun Xie

Shanxi Medical University

Suhui Wu ( $\square$ doctorwusuhui@163.com )

Third Hospital of Shanxi Medical University https://orcid.org/0000-0003-1674-4111

\section{Primary research}

Keywords: cervical cancer, drug resistance, cisplatin, ceRNA

Posted Date: July 1st, 2021

DOl: https://doi.org/10.21203/rs.3.rs-651847/v1

License: (c) (i) This work is licensed under a Creative Commons Attribution 4.0 International License.

Read Full License 


\section{Abstract}

Objective:: Cervical cancer (CC) is one of the most common malignant tumors in women. In order to identify the function between mRNA and non-coding RNA (ncRNA, including IncRNA,circRNA, miRNA) in CC DDP resistance, we analyzed its expression related to transcription profile and the RNA regulatory network between ncRNA and mRNA.

Methods: In this study, whole transcriptome high-throughput sequencing (RNA-sequencing) analysis was used to study the ncRNA profiles of parental SiHA cells and DDP-resistant SiHA/DDP cells. Conducted gene ontology (GO) and Kyoto Encyclopedia of Genes and Genomes (KEGG) pathway analysis, and analyzed mRNAs (DE) with significant differences in expression. Then, based on the authoritative cytoscape software v.3.7.2, drug resistance-related genes and signal transduction pathways, a ceRNA network combining IncRNA and mRNA was predicted and constructed. In addition, a constructed ceRNA regulatory pathway was randomly selected, namely Inc-AC010198.2 / hsa-miR-34b-3p / STC2, and verified by real-time qPCR, dual luciferase reporter gene system, and RNA pull-down assay. After transfection with si-Inc-AC010198.2 and DDP resistance, the changes in gene expression and biological function in SiHA and SiHA/DDP cells were further analyzed.

Results: Through bioinformatics and dual-luciferase reporter gene analysis, we found that IncAC010198.2 / miR-34b-3p / STC2 may be the mechanism by which SiHA / DDP cells are resistant to DDP compared to parent SiHA cells. After si-Inc-AC010198.2 is resistant to DDP after transfection, there are significant differences between SiHA/DDP and SiHA cells' downstream gene expression, the biological function of colony formation, invasion efficiency, and cell apoptosis.

Conclusions: Our study may provide new markers and potential mechanisms for CC DDP resistance, and discover some novel targets for reversing it.

\section{Introduction}

Cervical cancer (CC) caused by persistent human papillomavirus (HPV) infection is one of the most common malignant tumors in women worldwide and an important cause of death from cancer [1]. When first diagnosed, cancer sometimes progresses, especially in underdeveloped areas [2]. Cisplatin-based chemotherapy was included in the standard treatment plan for patients with advanced CC by the International Federation of Obstetrics and Gynecology (FIGO) [3]. However, cisplatin (DDP) resistance has complex mechanisms, such as DNA damage repair and activation of tumor cells $[4,5]$, reduction of apoptosis [6], target mutations or changes [7-9], and its complexity, Greatly limit the therapeutic effect. Overexpression of MDR-related proteins. However, the specific pathogenesis of CC is still unclear [10]. Therefore, further clarifying the mechanism of cervical cancer resistance to cisplatin can improve the clinical treatment effect of cervical cancer patients.

Non-coding RNA (ncRNA) is a type of RNA transcribed through regions of the human genome, including microRNA (miRNA) that does not code for protein, circular RNA (circRNA) and long non-coding RNA 
(IncRNA) [11]]. At least 75\% of the human genome is transcribed into ncRNA [12]. miRNA is a small single-stranded ncRNA, it consists of 19-25 nucleotides that regulates gene expression mainly by combining with sequence motifs located in the 3'-untranslated region (UTR) of mRNA transcripts [13]. LncRNA is a type of ncRNA with a length of more than 200 nucleotides, which is mainly transcribed by RNA polymerase II. Its characteristics are similar to messenger RNA, with a 5'cap and a 3'-poly(A) tail [14]. CircRNA is a new type of ncRNA with a closed-loop structure, which can participate in a series of biological and pathological processes like a sponge of miRNA, thereby regulating the progression of the disease [15]. In recent years, with the development of bioinformatics methods and high-throughput deep sequencing technology, it has been discovered that more and more ncRNAs play a key role in tumor development, including tumor cell proliferation, migration, metastasis and DDP resistance. Before transcription, transcription and after transcription, the expression level of ncRNAs is changed to regulate the expression of target genes to achieve tumor resistance [16]. In the past few years, competitive endogenous RNAs (ceRNA) have been shown to be a class of potential post-transcriptional regulators that can alter gene expression through miRNA-mediated mechanisms [17]. These ceRNAs, including various types of RNAs, such as mRNA, IncRNA, circRNA and pseudogenes, all have a common miRNA response element (MRE). They compete to bind miRNAs and regulate the expression of their target genes, thereby affecting tumor progression and resistance. Medicine [18].

Recently, it has been reported that the emerging ceRNA network and ncRNA are involved in cisplatin resistance in CC. For example, the significantly up-regulated miR-499a can promote the progression of CC and DDP resistance by targeting SOX6 [19]. Overexpressed miR-7-5p can increase the cisplatin resistance of CC cells by targeting BCL2 and PARP-1 to promote autophagy and inhibit cell DNA repair [20]. The highly expressed IncRNA HOXD-AS1 acts as a sponge for miR-130a-3p, up-regulating ZEB1, thereby enhancing the resistance of CC cells to DDP [21]. The up-regulation of IncRNA PCAT6 and miR-543/ZEB1 axis can regulate the DDP resistance of CC cells to cisplatin [22]. The over-expressed IncRNA NCK1-AS1 can reduce DDP-induced apoptosis by targeting the miR-134-5p/MSH2 axis, leading to the DDP resistance of CC to DDP [23]. The down-regulation of IncRNA CASC2 and miR-21/PTEN axis may play an important role in the DDP resistance of CC [24]. However, there is a lack of systematic evidence on the role of ceRNA in CC DDP resistance.

Herein, we use whole transcriptome sequencing technology to screen $\mathrm{CC}$ cells $(\mathrm{SiHA})$ and their paired DDP resistant cell pairs (SiHA/DDP) to find differentially expressed IncRNA, circRNA, miRNA and mRNA, and through quantitative real-time PCR (RT-QPCR). Through gene ontology (GO), the "Encyclopedia of Kyoto Protocol Genes and Genomes" (KEGG) approach analyzed significantly different expressed mRNAs regulated by circRNA / IncRNA-miRNA interactions in CC DDP resistance. Then, through the prediction of TargetScan and miRanda databases, combined with key signal transduction pathways and drug resistance-related genes, a ceRNA network of IncRNA, circRNA, miRNA and mRNA was predicted and constructed. Finally, a ceRNA pathway was selected and verified by RNA pull-down assay, RT-QPCR and dual luciferase reporter gene system. Here, our findings may explore new evidence of potential molecular biomarkers and their co-expression network in CC DDP resistance, and discover some novel targets for reversing its DDP resistance. 


\section{Materials And Method}

Cell culture

The human CC cell line SiHA was purchased from the Shanghai Chinese Academy of Sciences Cell Bank (Shanghai, China), and drug resistance was induced by continuously increasing the drug concentration for more than 8 months. Cell lines with DDP resistance (SiHA / DDP) were screened. Cancer cells are cultured in RPMI 1640 medium, and add $100 \mathrm{U} / \mathrm{mL}$ streptomycin ,100 pg/mL penicillin,and 10\% FBS (Gibco). Culture in a cell incubator $\left(37^{\circ} \mathrm{C}, 5 \% \mathrm{CO}_{2}\right)$. Use lipofectamine2000 packaged $75 \mathrm{nM}$ SiRNA / miRNA plasmid or mixed negative control (Ribobio) to transfect SiHA / DDP cells, collect the transfected cells 48 hours later, use a microplate reader to record the absorbance in the $490 \mathrm{~nm}$ region. The IC50 value is calculated by drawing a dose-response curve.

\section{Cell counting kit 8 assay}

Inoculate attached $\mathrm{SiHA}$ and SiHA/DDP cells $\left(1 \times 10^{4}\right.$ cells/well) into a 96-well plate, incubate for 24 hours, and then treat the cells with $100 \mu$ l of medium containing different concentrations of DDP (sigma) $(0.01 \mu \mathrm{M}), 0.1 \mu \mathrm{M}, 1 \mu \mathrm{M}, 3 \mu \mathrm{M}, 10 \mu \mathrm{M}, 30 \mu \mathrm{M}, 100 \mu \mathrm{M}, 300 \mu \mathrm{M})$. After incubating for another 24 hours, replace the medium in each well with $100 \mu \mathrm{l}$ of the medium of the Cell Counting Kit-8 (CCK-8, GLPBIO, USA) containing $0.5 \mathrm{mg} / \mathrm{mL}$ WST-8, and continue Incubate in a cell culture incubator for $2 \mathrm{~h}\left(37^{\circ} \mathrm{C}\right)$. Finally, use a microplate reader to record the absorbance in the $450 \mathrm{~nm}$ region. Each experiment was repeated three times.

\section{RNA extraction and quality control}

The total RNA of two adherent cells was extracted by the Trizol method. After the RNA precipitate was dissolved, the RNA sample concentration was preliminarily detected by Qubit ${ }^{2} 2.0$ fluorometer, and the RNA sample was further diluted with RNase-free water, electrophoresed on a $1 \%$ agarose gel at $180 \mathrm{~V}$ for 16 minutes, and Agilent Bioanalyzer 2100 The RNANano6000 of the system (Agilent Technologies, CA, USA) detects the integrity of RNA samples and whether there are other impurities/genomic contamination.

\section{Library construction and sequencing}

Utilizing the structural features of most eukaryotic mRNAs with polyA tails, the mRNAs with polyA tails are enriched and purified by Oligo (dT) magnetic beads, and then the resulting mRNAs are randomly interrupted by using divalent cations in NEB Fragmentation Buffer Finally, build the library according to the chain-specific library method to obtain a miRNA library for detecting the sequence information of miRNA, and a chain-specific library for removing ribosomal RNA for detecting the sequence information of mRNA, circRNA and IncRNA. The qualified library is pooled according to the effective concentration and target data volume requirements, and then the sequencing by synthesis (Sequencing by Synthesis) is performed on the Illumina Hiseq 4000 platform. 


\section{Analysis of GO and KEGG pathways}

GO (http://geneontology.org/) analysis is a functional analysis that uses clusterProfiler software to enrich the differential gene set and uses the principle of hypergeometric distribution to find specific biological functions or biological pathways that are significantly related to differential genes [25].

The KEGG database (https://www.kegg.jp/kegg/) integrates genomic, chemical, and system function information [26]. The Pathway database integrates current molecular interaction networks and contains more advanced gene function information. Use clusterProfiler software to calculate the enrichment of differential genes in the KEGGPathway entry, so as to find a significant enrichment signal pathway.

\section{Competitive endogenous RNA network}

According to the ceRNA hypothesis, if a certain LncRNA can regulate miRNA, and the miRNA can regulate one or more targeted mRNAs, the LncRNA may be used as a ceRNA to regulate mRNA. Based on the results obtained by analyzing high-throughput sequencing data, the two search platforms DIANALncBase V2 (http://www.microma.gr/LncBase) and miRcode (http://www. mircode.org/mircode) are used to predict For miRNAs that bind to LncRNA, use miRTarBase (http://www.mirtarbase.mbc.ncm.edu.tw) platform to retrieve miRNA-targeted mRNA. Cytoscape software v.3.7.2 (San Diego, California, USA) was used to construct the ceRNA network.

\section{Real-time quantitative polymerase chain reaction}

Follow the steps in the Thermo Scientific GeneJET RNA Purification Kit (Thermo Scientific, USA) instructions to extract two total cell RNA samples, and use HiScriptllIRTSuperMix (Vazyme Biotech Co., $L t d)$ to reverse transcribed them into cDNA. With $\beta$-actin as the internal reference gene, the ABIViiA7 realtime PCR system (Thermo Lifetech) was used to detect the relative expression of genes, and three RTqPCR reactions were performed for each gene. The data was expressed in the form of $2^{-\Delta \Delta C t}$. Use the software primerpremier5.0 to design primers and select the primer sequence with the highest score in the result. The primer sequences are listed in Table 1.

Table 1: Primer sequences of genes 


\begin{tabular}{|c|c|}
\hline Gene & Primer \\
\hline \multirow[t]{2}{*}{ AC010198.2 } & F®5'- GCCGCGATTTTTGTGTCCAA -3' \\
\hline & R囚5'- GACACTTCTCGAGGGCGCTT -3’ \\
\hline \multirow[t]{2}{*}{ STC2 } & F囚5'- GGGTGTGGCGTGTTTGAATG -3' \\
\hline & R囚5'- CTTGAGGTAGCATTCCCGCT -3' \\
\hline \multirow[t]{2}{*}{ GAPDH } & F®5'-ACCACAGTCCATGCCATCAC -3' \\
\hline & R囚5'-TCCACCACCCTGTTGCTGTA -3' \\
\hline \multirow{2}{*}{$\begin{array}{l}\text { hsa-miR-34b- } \\
3 p\end{array}$} & F『5'-AGGCAGTGTAGTTAGCTGATTGC -3' \\
\hline & R囚5'-TGGTGTCGTGGAGTCG -3' \\
\hline \multirow[t]{3}{*}{ U6 } & $\begin{array}{l}\text { RT5'-CTCAACTGGTGTCGTGGAGTCGGCAATTCAGTTGAGTTTTTTTTTTTTTTTAG } \\
-3^{\prime}\end{array}$ \\
\hline & F『5'- GCTTCGGCAGCACATATACTAAAAT -3' \\
\hline & R囚5'- CGCTTCACGAATTTGCGTGTCAT -3' \\
\hline
\end{tabular}

\section{Western Blot}

The CC cells were lysed with RIPA lysis solution, and the total protein was extracted with a protein extraction kit, the protein concentration was measured by the BCA method, and the protein was separated by $10 \%$ SDS-PAGE under voltage. Then use a constant current of $300 \mathrm{~mA}$ to transfer the protein to the PVDF membrane and place it in the TBST solution for use. Use pre-prepared $5 \%$ skimmed milk to seal for 1 hour. Then, add the PVDF membrane to the prepared primary antibody solution and incubate overnight at $4^{\circ} \mathrm{C}$ (1:1,000 dilution; Abcam). Then, add the secondary antibody solution (1:500) and incubate for 2 hours. Next, after dropping ECL developer solution on the film for exposure and development, use ImageJ software to calculate the band gray value for data analysis. Use $\beta$-actin as an internal reference.

\section{RNA pull-down and luciferase reporter assay}

miR-34b-3p-WT or miR-34b-3p-Mut is synthesized by Sangon Biotech (Shanghai, China), incubated with biotin-labeled probes for 4 hours, added to the sample at a ratio of 2:1, and incubated with 100ul magnetic beads for 30 minutes, The magnetic stand separates the magnetic beads. For the operation steps of RNA pull-down, please refer to Zhu 2019 [27]. Next, perform real-time qPCR verification on the eluted RNA-binding protein complex.

Construct the pGL3-AC010198.2 and pGL3-3'UTRCDNA vector containing the binding fragment (AC010198.2 and 3'UTRcDNA of STC2 chemically synthesized), and the Renilla luciferase reporter gene as a control. The SiHA cells grown to $80 \%$ were trypsinized to prepare a cell suspension. The cell suspension (cell number approximately $4 \times 10^{5}$ ) was seeded in a 24 -well culture plate, and then miR-34b- 
$3 p$ mimic or negative control $(150 \mathrm{nM})$ was transfected into the cells using lipofectamine 3000 . Observe the transfection efficiency under a fluorescence microscope 24 hours after transfection. Use cell lysate to lyse cells, pipette $100 \mu \mathrm{L}$ of cell lysate into Lockwell maxisorp detection plate, use dual luciferase reporter gene detection kit (Promega, USA) to detect fluorescence value (RLU), and divide by Renilla luciferase assay Obtain the fluorescence value (RLU), and obtain the activation degree of the reporter gene between different samples. The same experimental method is applicable to verify the relationship between AC010198.2 and miR-34b-3p, miR-34b-3p and STC2.

\section{Colony formation, cell invasion assays}

For the determination of colony formation, use fresh MEM medium 1:1 to prepare $0.7 \%$ agarose and spread it on the culture plate, add about $1 \times 10^{4} / \mathrm{cm}^{2}$ cells per well to the cell culture plate, and set 3 parallel controls. Place them in a $37^{\circ} \mathrm{C}, 5 \% \mathrm{CO} 2$ constant temperature incubator and culture them with Giemsa solution (Beyotime, China) for staining. Take out the petri dish and place it under an inverted microscope to count. A clump of $\geq 50$ cells is counted as 1 cell colony and photographed for storage. Calculate the plate clone formation rate. All experiments were repeated three times.

The upper surface of the membrane at the bottom of the Transwell cell was coated with $50 \mathrm{mg} / \mathrm{L}$ Matrigel ( $1: 8$ dilution). Take different groups of cell suspensions $\left(10^{6} \mathrm{cells} / \mathrm{mL}\right) 200 \mu \mathrm{L}$ and add them to the transwell chamber. After 24 and 48 hours of culture in an incubator, they were stained with $0.1 \%$ crystal violet, and the cells were counted and photographed under a microscope. Three independent replicate experiments were performed.

\section{Cell apoptosis}

The experiment was divided into four groups. The cells were cultured in a six-well plate, digested with trypsin without EDTA, and centrifuged at $2000 \mathrm{rpm}$ for 5 minutes at room temperature to collect the cells. After adding $5 \mu$ of Annexin V-FITC and mixing, it was detected by FACSCalibur flow cytometer according to standard procedures, and 10,000 cells were counted to analyze the ratio of apoptosis and necrosis.

\section{Statistical Analysis}

Use SPSS22.0 software and GraghPadPrism7.0 to analyze all experimental data of at least three independent experiments. In transcriptome sequencing analysis, differences in fold change (FC) $\geq 2$ or $\leq 0.5$ and $p<0.05$ were considered to be statistically significant. The expression level of each circRNA is expressed as a fold change as $2-\Delta \Delta \mathrm{Ct}$. The Student'st test was used to analyze the difference between the two groups, and the significance was defined as $p<0.05$.

\section{Results}

\section{Morphological characteristics and drug sensitivity assay in vitro}


Observed by an optical microscope, SiHA/DDP cells appear to be polygonal, while SiHA cells appear to be spindle-shaped and elongated (Figure 1-A). The increased pseudopodia formation in SiHA/DDP cell lines may promote the migration and invasion of $\mathrm{CC}$ cells.

The resistance was confirmed by comparing the $50 \%$ inhibitory concentration (IC50) value of drugresistant SiHA/DDP with the 50\% inhibitory concentration (IC50) value of the sensitive SiHA cell line. As shown in Figure 1-B, after 24 hours of incubation with different concentrations of DDP, the IC50 values of SiHA/DDP and SiHA cells were $212.78 \pm 17.89 \mu \mathrm{M}$ and $27.87 \pm 1.72 \mu \mathrm{M}$, respectively, an increase of 7.63 times. The results confirmed that the DDP-resistant CC cells were more resistant to DDP than the matched parental DDP-sensitive cells.

\section{Expression profile of circRNAs, IncRNAs, miRNAs and mRNAs}

The whole transcriptome sequencing data (circRNA, mRNA, IncRNA, miRNA) were obtained from the two sets of data through the IlluminaHiseq platform. We analyzed the differential expression (DE) ncRNA and mRNA of three pairs of DDP-resistant and DDP-sensitive CC strains with cut-off fold change $\geq 2$ or $\leq 0.5$, $p<0.05$ and false discovery rate $(F D R)<0.05$. The volcano map (Figure $2-A$ ) and heat map (Figure $2-B$ ) are used to show the DEncRNA and mRNA in the sample. The results showed that there were 2664 DElncRNAs (1309 up-regulated and 1355 down-regulated), 551 circRNAs (126 up-regulated and 425 down-regulated), 82 DEmiRNAs (38 up-regulated and 44 down-regulated) and 4790 mRNAs (2582 upregulated and 2208 down-regulated) .

As shown in Table 2, the IncRNA with the most up-regulated expression is Inc-SLC3A2 with a 20-fold change, and the IncRNA with the most down-regulated expression is LINC00852 with a 16-fold change. In addition, the most up-regulated circRNA, miRNA and mRNA were hsa_circRNA_05436 (a 24-fold change), hsa-novel-105 mature (a 13-fold change) and MORF4L2 (a 19-fold change). The most down-regulated circRNA, miRNA and mRNA are hsa_circRNA_02887 (a 24-fold change), hsa-novel-4 mature (a 10-fold change) and HLA-A (a 19-fold change). Table S1-S3 lists the top 20 up-regulated and down-regulated IncRNA, circRNA and mRNA. Table S4 lists the top 10 up-regulated and down-regulated miRNAs.

Table 2: Statistical analysis of all DE ncRNA and mRNA

\begin{tabular}{|c|c|c|c|c|c|}
\hline $\begin{array}{l}\text { DE } \\
\text { RNAs }\end{array}$ & $\begin{array}{l}\text { Total } \\
\text { No. }\end{array}$ & $\begin{array}{l}\text { No. of } \\
\text { Upregulated }\end{array}$ & $\begin{array}{l}\text { No. of } \\
\text { Downregulated }\end{array}$ & $\begin{array}{l}\text { The Most } \\
\text { Upregulated } \\
\text { (Fold Change) }\end{array}$ & $\begin{array}{l}\text { The Most } \\
\text { Downregulated (Fold } \\
\text { Change) }\end{array}$ \\
\hline
\end{tabular}

\begin{tabular}{cccccl}
\hline IncRNA & 2664 & 1309 & 1355 & SLC3A2 (20.44) & LINC00852 (16.36) \\
\hline circRNA & 551 & 126 & 425 & $\begin{array}{l}\text { hsa_CircRNA_05436 } \\
(24.91)\end{array}$ & $\begin{array}{l}\text { hsa_circRNA_02887 } \\
(24.83)\end{array}$ \\
\hline miRNA & 82 & 38 & 44 & $\begin{array}{l}\text { hsa-novel-105- } \\
\text { mature }(13.64)\end{array}$ & $\begin{array}{l}\text { hsa-novel-4-mature } \\
(10.15)\end{array}$ \\
\hline mRNA & 4790 & 2582 & 2208 & MORF4L2 (19.93) & HLA-A (19.28) \\
& & & & Page 8/21
\end{tabular}




\section{Bioinformatics analysis in ceRNA network}

According to analysis, we further constructed circRNA-miRNA-mRNA network and IncRNA-miRNA-mRNA network. The GO and KEGG pathways of ncRNA host genes were analyzed to further analyze DE mRNA and speculate its potential functions. GO analysis showed that these DEmRNAs in the ncRNAs network are mainly related to the positive regulation of type XIV collagen trimer (cell component), tolerance induction (biological process), and keratin filament binding (molecular function) (Figure 3-A). Enrichment analysis of pathways by KEGG showed that there were 21 pathways in the up-regulated circRNA and 35 pathways were enriched in the down-regulated circRNA. Among them, glycosphingolipid biosynthesisglobo series and linoleic acid metabolism signal transduction pathway are the most abundant (Figure (3B)).

\section{A ceRNA network constructed based on important genes related to DDP resistance}

According to reports, CircRNA can act as a miRNA sponge to manipulate gene expression, thereby playing a role in the development of cancer. We predicted potential target miRNAs and used miRanda and TargetScan databases to obtain all DE circRNA and IncRNA. We further filtered the key DE mRNA closely related to drug resistance, and obtained a more concise and effective ceRNA network.

Based on the sequencing data, we screened and established some IncRNA-miRNA-mRNA and circRNAmiRNA-mRNA interaction networks, and used Cytoscape to illustrate them (Figure 4). Then 317 mRNAs were selected in the IncRNA-miRNA-mRNA network, which participated in 11 pathways, for example, glycosphingolipid biosynthesis-globo series and linoleic acid metabolism signaling pathway, chemical carcinogenesis, cytochrome P450, PI3K-Akt signaling pathway. In addition, 121 mRNAs selected in the circRNA-miRNA-mRNA network participate in 9 pathways, such as $A B C$ transporter, Wnt/ $\beta$-catenin signaling pathway, drug metabolism-cytochrome P450, PI3K-Akt signaling pathway, etc. Then, we constructed the IncRNA/cirRNA-miRNA-mRNA network based on the selected mRNA. There are 2989 IncRNA-miRNA-mRNA pathways constructed, including 134 IncRNA, 37 miRNA and 317 mRNA (Figure 4A). In addition, 4,123 cirRNA-miRNA-mRNA pathways were established, including 498 circRNA, 24 miRNA and 121 mRNA (Figure 4-B). Based on the IncRNA-miRNA-mRNA network, it is expected that AC010198.2 can bind to a series of miRNAs with known functions, indicating that they have a potential role in the DDP resistance of $\mathrm{CC}$. These results indicate that, considering the complexity between ncRNA and mRNA, these RNA interactions may be critical to the pathogenesis of CCDDP resistance.

\section{AC010198.2/hsa-miR-34b-3p/STC2 pathway was selected to verify the feasibility of the constructed IncRNA/miRNA/mRNA network}

In SiHA cells, we randomly selected the IncRNA-AC010198.2/hsa-miR-34b-3p/STC2 pathway to verify the feasibility of the ceRNA network. Firstly, the expression levels of AC010198.2, miR-34b-3p and STC2 in DDP-sensitive and DDP-resistant CC cell lines and tissues were checked. The results showed that compared with DDP-resistant CC cell lines (Figure 5-A and 5-C) and tissues (Figure 5-E and 5-G), the mRNA levels of AC010198.2 and STC2 genes were significantly increased, and STC2 The expression of 
the protein increased (Figure 5-D). In the anti-DDP CC cell line (Figure 5-B) and tissues (Figure 5-F), the expression level of miR-34b-3p decreased. This is consistent with the results in RNA-seq. Next, we constructed small interfering RNA (siRNA) and transfected it into the SiHA/DDP cell line. Compared with siHA/DDP and SiHA/DDP-NC, the expression of miR-34b-3p and STC2 after transfection of siAC010198.2 by qPCR method has the highest inhibitory effect (Figure (5-AD). In SiHA In the /DDP cell line, when siRNA down-regulated AC010198.2, the expression of STC2mRNA and protein was significantly reduced, which can be rescued by inhibiting the expression of miR-34b-3p.

In order to analyze the overall survival rate of miR-34b-3p and STC2 with cervical squamous cell carcinoma and internal cervical adenocarcinoma (CESC), we used KAPLA and GEPIA to perform statistical analysis through the TCGA database. We found that the highly expressed miR-34b-3p indicated longer survival time in CESC (Figure 5-H), and the opposite association with STC2 was observed (Figure $5-I)$. The bioinformatics prediction tool further proved that miR-34b-3p targets AC010198.2 and 3'UTR of STC2 with complementary binding sites (Figure 5-M-N). A luciferase reporter plasmid containing wild-type (WT) and mutant (MUT) AC010198.2 and STC2 was constructed. Obviously, co-transfection of luciferase reporter plasmid containing WT-AC010198.2 (MUT-AC010198.2) or WT-STC2 (MUT-STC2) and miR-34b-3p mimics into SiHA cells resulted in reporter activity Lower (Figure 5-KL). The above results indicate that miR-34b-3p targets AC010198.2 and 3'UTR of STC2 through molecular binding. Subsequently, compared with the control miR-34b-3p-MUT-bio probe, the RNA pull-down assay using the biotin-labeled miR-34b-3pWT-bio probe can increase the expression of AC010198.2 or STC2 (Figure $5-J$ ). In summary, the above results confirmed the AC010198.2/hsa-miR-34b-3p/STC2 axis and proved its role in CC DDP resistance.

\section{The function of AC010198.2 in CC cells After Si-AC010198.2 transfection}

After si-AC010198.2 was transfected and DDP was treated with IC50, the gene expression and biological function changes of SiHA and SiHA/DDP cells were further analyzed to analyze the synergistic effect of AC010198.2 and DDP on tumor cells. We further explored whether si-AC010198.2 can inhibit the formation, migration and apoptosis of colony SiHA/DDP cells. Compared with SiHA/DDP and SiHA/DDPNC cells, SiHA/DDP cells transfected with si-AC010198.2 significantly inhibited migration and invasion (Figure 6-A-D). In summary, these findings confirm that high expression of AC010198.2 promotes the colony and motility of SiHA/DDP cells, which is a typical feature of cancer stem cells. At the same time, we measured the apoptosis of the four groups of cells by flow cytometry after si-AC010198.2 was transfected and placed DDP, as shown in Figure 6-E. As shown in Figure 6-F, statistical analysis of the number of apoptosis of the four groups of cells showed that after processing the SiHA/DDP cells of siAC010198.2, more apoptosis was promoted. Compared with SiHA/DDP cells, IC50-treated DDP significantly promoted the apoptosis of SiHA, while the low expression of AC010198.2 can promote the sensitivity to DDP.

\section{Discussions}


DDP resistance is an important challenge encountered in the treatment of CC [4]. In the past few decades, despite extensive research, the specific mechanism of drug resistance has not yet been elucidated. More and more evidences indicate that ncRNAs may play a key role in the resistance of cancer [28]. For instance, Huang et al. [29] reported that circAKT3 can act as the cavernous body of miR-198, promote the expression of PIK3R1 in gastric cancer and enhance the resistance to DDP. Zhu et al. [30] found that circPVT1 promotes adriamycin and DDP resistance in OS cells by regulating ABCB1. Su et al. [31] showed that hypoxia circELP3 can promote the proliferation of bladder cancer cells and DDP resistance, and is related to the prognosis. Shao et al. [32] screened the circRNA expression profile in pancreatic cancer cell lines through transcriptome high-throughput sequencing analysis and found that two circRNAs may be related to gemcitabine resistance in pancreatic ductal adenocarcinoma. Liu et al. [33] reported that circEIF6 can promote canceration of thyroid cancer cells and enhance DDP resistance by regulating miR144-3p/TGF-a. Long et al [34] reported that the GAS5-E2F4-PARP1-MAPK axis inhibited the progression of epithelial ovarian cancer and DDP resistance and resistance. Luo et al. [35] reported that the IncRNAHNF1A-AS1 axis affects the cisplatin resistance of cervical cancer cells. However, no research on ncRNA dysregulation in $\mathrm{CC}$ resistance has been reported.

Herein, we first used transcriptome high-throughput sequencing analysis to compare the expression profiles of ncRNAs between DDP-resistant and sensitive CC cell lines (Siha/DDP and Siha). Subsequently, we used qRT-PCR analysis to detect the expression levels of IncRNAs in Siha/DDP and Siha cells and tissues to verify the sequencing data. After that, bioinformatics analysis including GO, KEGG pathway analysis and circRNA/miRNA/mRNA and IncRNA/miRNA/mRNA networks were carried out to study the biological functions and mechanisms of these ncRNAs in CC resistance. Finally, after transfection of siAC010198.2 and IC50 treatment of DDP, the biological functions of SiHA and SiHA/DDP cells were further analyzed to analyze the synergistic effect of AC010198.2 and DDP on tumor cells. It shows that AC010198.2 can significantly affect the cloning, invasion and apoptosis of SIHA/DDP after interference (Fig. 6), and it has been confirmed that AC010198.2 has a key role in regulating the DDP resistance of SiHA cells.

From the sequencing data, we found that 2989 IncRNA/miRNA/mRNA pathways were constructed, including $134 \mathrm{IncRNA}, 37 \mathrm{miRNA}$ and $317 \mathrm{mRNA}$, and 4,123 cirRNA/miRNA/mRNA/mRNA pathways were established, including 498 circRNA, 24 miRNAs and 121 mRNAs $(F C \geq 2$ or $\leq 0.5$ and $p<0.05)$. There are 71 up-regulated and 63 down-regulated IncRNAs, 105 up-regulated and 393 down-regulated cirRNAs, 29 up-regulated and 32 down-regulated miRNAs, and 217 up-regulated and 221 down-regulated mRNAs. It may be related to the DDP resistance in the CC unit. Among them, the top $10 \mathrm{ncRNAs}$ regulated by dys were selected to verify the reliability of RNA-seq using qRT-PCR. We found that the RNA-seq data is very consistent with qRT-PCR. In addition, we found that compared with Siha/DDP cells, the circRNA (hsa_circRNA_05436[+ 24.91], hsa_circRNA_02887[-24.83]) and IncRNA (SLC3A2[+ 20.44], LINC00852[-16.36] with the greatest difference in expression of siRNA/siRNA/DDP cells ). .

GO enrichment analysis and KEGG pathway analysis show that certain gene symbols are involved in the regulation of biological processes, cell components and molecular functions. Glycosphingolipid

Page $11 / 21$ 
biosynthesis-globo series and linoleic acid metabolism signaling pathways, chemical carcinogenesis, metabolism of cytochrome P450 heterologous organisms, PI3K-Akt signaling pathway and other 11 pathways are in circRNA and 9 pathways, including $A B C$ transporter, Wnt/ $\beta$-catenin signaling pathway is not only that, PI3K-Akt signaling pathway, drug metabolism-cytochrome P450, is significantly abundant in down-regulated circRNAs.

More and more evidences show that circRNAs can regulate miRNA expression at the transcription or posttranscriptional level [36-38]. Chen et al. [39] reported that circRNA_100290 played a role in regulating the miR-29 family and regulating the progression of oral cancer. Cheng et al. [40] found that circTP63 affects the progression of lung squamous cell carcinoma by regulating miR-873-3p. Zheng et al. [41] showed that circHIPK3 can bind to nine miRNAs to regulate target gene expression. Given that miRNAs play a key role in the DDP resistance of CC [42], some IncRNAs may participate in DDP resistance by interacting with miRNAs. Since we identified IncRNA-AC010198.2 with significantly different expressions in this study, obtaining more information about the miRNA network of circRNA is very meaningful for our future research. This helps to better understand its upstream and downstream miRNA targets as potential CC biomarkers and DDP resistance targets.

\section{Conclusion}

In conclusion, our study revealed for the first time the expression profile of differentially expressed circRNA in DDP-resistant and sensitive CC cell lines. We analyzed the GO and KEGG pathways of DEncRNA, and constructed the AC010198.2/hsa-miR-34b-3p/STC2 interaction network, which helps us understand the mechanism of CCDDP resistance and find some novel targets to reverse it.

\section{Declarations}

\section{consent for publication}

All authors read the final manuscript and consent for publication.

\section{Competing interests}

The authors declare no competing interests.

\section{Funding}

This work was supported by a grant from the Applied Basic Research Programs of Science and Technology Commission Foundation of Shanxi Province (No.201901D111402)『and Scientific Research Project of Health Commission of Shanxi Province(No.2021151).

\section{Authors' contributions}


Suhui Wu and Huimin Lv participated in the study design and statistical analysis. Huimin Lv and Shanshan Jin carried out the cell experiments. Binbin Zou carried out the bioinformatic analysis. Yuxiang Liang helped to correct the results of analysis. Jun Xie provided the experimental platform.

\section{Availability of data and materials}

Availability of data and supporting materials section: "Please contact author for data requests. Please contact the email:doctorwusuhui@163.com

\section{Acknowledgements}

All authors read and approved the final manuscript.

\section{Ethical Approval}

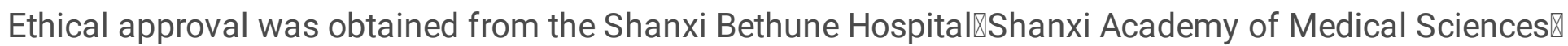
Tongji Shanxi Hospital凹Third Hospital of Shanxi Medical University.

\section{References}

[1] Bray, F., et al., Global cancer statistics 2018: GLOBOCAN estimates of incidence and mortality worldwide for 36 cancers in 185 countries. CA Cancer J Clin, 2018. 68(6): p. 394-424.

[2] Shi, J.F., K. Chen Jf Fau - Canfell, X.-X. Canfell K Fau - Feng, J.-F. Feng Xx Fau - Ma, Y.-Z. Ma Jf Fau Zhang, F.-H. Zhang Yz Fau - Zhao, R. Zhao Fh Fau - Li, L. Li R Fau - Ma, Z.-F. Ma L Fau - Li, J.-B. Li Zf Fau Lew, Y. Lew Jb Fau - Ning, Y.-L. Ning Y Fau - Qiao, and Y.L. Qiao, Estimation of the costs of cervical cancer screening, diagnosis and treatment in rural Shanxi Province, China: a micro-costing study[J]. (1472-6963 (Electronic)).

[3] Small, W.J.A.-O., et al., Cervical cancer: A global health crisis. (1097-0142 (Electronic)).

[4] Henrique, R., S.P. Nunes, and C. Jerónimo, MSH2 Expression and Resistance to Cisplatin in Muscleinvasive Bladder Cancer: A Mix of Progress and Challenges[J]. Eur Urol, 2019. 75(2): p. 251-252.

[5] Li, Y., S. Zhang, Y. Wang, J. Peng, F. Fang, and X. Yang, MLH1 enhances the sensitivity of human endometrial carcinoma cells to cisplatin by activating the MLH1/c-Abl apoptosis signaling pathway[J]. BMC Cancer, 2018. 18(1): p. 1294.

[6] Stordal, B. and M. Davey, Understanding cisplatin resistance using cellular models[J]. IUBMB Life, 2007. 59(11): p. 696-9.

[7] Bompiani, K.M., C.Y. Tsai, F.P. Achatz, J.K. Liebig, and S.B. Howell, Copper transporters and chaperones CTR1, CTR2, ATOX1, and CCS as determinants of cisplatin sensitivity[J]. Metallomics, 2016. 8(9): p. 95162. 
[8] Lee, Y.Y., C.H. Choi, I.G. Do, S.Y. Song, W. Lee, H.S. Park, T.J. Song, M.K. Kim, T.J. Kim, J.W. Lee, D.S. Bae, and B.G. Kim, Prognostic value of the copper transporters, CTR1 and CTR2, in patients with ovarian carcinoma receiving platinum-based chemotherapy[J]. Gynecol Oncol, 2011. 122(2): p. 361-5.

[9] Zhu, H., H. Luo, W. Zhang, Z. Shen, X. Hu, and X. Zhu, Molecular mechanisms of cisplatin resistance in cervical cancer[J]. Drug Des Devel Ther, 2016. 7(10): p. 1885-95.

[10] Ulitsky, I. and D.P. Bartel, lincRNAs: genomics, evolution, and mechanisms. (1097-4172 (Electronic)).

[11] Agostini, M., C.A.-O. Ganini, E. Candi, and G. Melino, The role of noncoding RNAs in epithelial cancer[J]. (2058-7716 (Print)).

[12] Beermann, J., et al., Non-coding RNAs in Development and Disease: Background, Mechanisms, and Therapeutic Approaches. (1522-1210 (Electronic)).

[13] Lai, E.C., Micro RNAs are complementary to 3' UTR sequence motifs that mediate negative posttranscriptional regulation[J]. (1061-4036 (Print)).

[14] Ulitsky, I. and D.P. Bartel, lincRNAs: genomics, evolution, and mechanisms[J]. (1097-4172 (Electronic)).

[15] Chen, B. and S. Huang, Circular RNA: An emerging non-coding RNA as a regulator and biomarker in cancer[J]. (1872-7980 (Electronic)).

[16] Hansen, T.B., et al., Natural RNA circles function as efficient microRNA sponges. (1476-4687 (Electronic)).

[17] Hansen, T.B., B.H. Jensen Ti Fau - Clausen, J.B. Clausen Bh Fau - Bramsen, B. Bramsen Jb Fau Finsen, C.K. Finsen B Fau - Damgaard, J. Damgaard Ck Fau - Kjems, and J. Kjems, Natural RNA circles function as efficient microRNA sponges[J]. (1476-4687 (Electronic)).

[18] Du, H. and Y. Chen, Competing endogenous RNA networks in cervical cancer: function, mechanism and perspective[J]. (1029-2330 (Electronic)).

[19] Yang, F., et al., MicroRNA-7-5p Promotes Cisplatin Resistance of Cervical Cancer Cells and Modulation of Cellular Energy Homeostasis by Regulating the Expression of the PARP-1 and BCL2 Genes. (1643-3750 (Electronic)).

[20] Yang, F., L. Guo, Y. Cao, S. Li, J. Li, and M. Liu, MicroRNA-7-5p Promotes Cisplatin Resistance of Cervical Cancer Cells and Modulation of Cellular Energy Homeostasis by Regulating the Expression of the PARP-1 and BCL2 Genes[J]. (1643-3750 (Electronic)).

[21] Chi, C., M. Mao, Z. Shen, Y. Chen, J. Chen, and W. Hou, HOXD-AS1 Exerts Oncogenic Functions and Promotes Chemoresistance in Cisplatin-Resistant Cervical Cancer Cells[J]. Hum Gene Ther, 2018. 
[22] Ma, Z.A.-O.X., G. Gu, W. Pan, and X. Chen, LncRNA PCAT6 Accelerates the Progression and Chemoresistance of Cervical Cancer Through Up-Regulating ZEB1 by Sponging miR-543[J]. (1178-6930 (Print)).

[23] Feng, Y., et al., Modulation of CASC2/miR-21/PTEN pathway sensitizes cervical cancer to cisplatin. Arch Biochem Biophys, 2017. 623-624: p. 20-30.

[24] Ashburner, M., et al., Gene ontology: tool for the unification of biology. The Gene Ontology Consortium. (1061-4036 (Print)).

[25] The Gene Ontology Resource: 20 years and still GOing strong. (1362-4962 (Electronic)).

[26] Kanehisa, M., Y. Sato, M. Furumichi, K. Morishima, and M. Tanabe, New approach for understanding genome variations in KEGG[J]. (1362-4962 (Electronic)).

[27] Zhu, H., H. Luo, W. Zhang, Z. Shen, X. Hu, and X. Zhu, Molecular mechanisms of cisplatin resistance in cervical cancer[J]. (1177-8881 (Electronic)).

[28] Hua, X., Y. Sun, J. Chen, Y. Wu, J. Sha, S. Han, and X. Zhu, Circular RNAs in drug resistant tumors[J]. (1950-6007 (Electronic)).

[29] Huang, X., et al., Circular RNA AKT3 upregulates PIK3R1 to enhance cisplatin resistance in gastric cancer via miR-198 suppression. (1476-4598 (Electronic)).

[30] Kun-Peng, Z., M. Xiao-Long, and Z. Chun-Lin, Overexpressed circPVT1, a potential new circular RNA biomarker, contributes to doxorubicin and cisplatin resistance of osteosarcoma cells by regulating ABCB1[J]. (1449-2288 (Electronic)).

[31] Su, Y., et al., Hypoxia-elevated circELP3 contributes to bladder cancer progression and cisplatin resistance. (1449-2288 (Electronic)).

[32] Shao, F., M. Huang, F. Meng, and Q. Huang, Circular RNA Signature Predicts Gemcitabine Resistance of Pancreatic Ductal Adenocarcinoma[J]. (1663-9812 (Print)).

[33]. Liu, F., et al., Circular RNA EIF6 (Hsa_circ_0060060) sponges miR-144-3p to promote the cisplatinresistance of human thyroid carcinoma cells by autophagy regulation. (1945-4589 (Electronic)).

[34]. Xiaoran Long, et al. Long non-coding RNA GAS5 inhibits DDP-resistance and tumor progression of epithelial ovarian cancer via GAS5-E2F4-PARP1-MAPK axis. J Exp Clin Cancer Res . 2019, 38(1):345.

[35]. Xiaoqiong Luo, et al. Exosomal IncRNA HNF1A-AS1 affects cisplatin resistance in cervical cancer cells through regulating microRNA-34b/TUFT1 axis. Cancer Cell Int . 2019 Dec 3;19:323.

[36]. Kristensen, L.A.-O., et al., The biogenesis, biology and characterization of circular RNAs. (1471-0064 (Electronic)). 
[37]. Qu, S., et al., The emerging landscape of circular RNA in life processes. (1555-8584 (Electronic)).

[38]. Qi, X., et al., ceRNA in cancer: possible functions and clinical implications. (1468-6244 (Electronic)).

[39]. Chen, L., et al., circRNA_100290 plays a role in oral cancer by functioning as a sponge of the miR-29 family. (1476-5594 (Electronic)).

[40]. Cheng, Z., et al., circTP63 functions as a ceRNA to promote lung squamous cell carcinoma progression by upregulating FOXM1. (2041-1723 (Electronic)).

[41]. Zheng, Q., et al., Circular RNA profiling reveals an abundant circHIPK3 that regulates cell growth by sponging multiple miRNAs. (2041-1723 (Electronic)).

[42]. Yu, M., et al., MicroRNA-218 regulates the chemo-sensitivity of cervical cancer cells through targeting survivin. (1179-1322 (Print)).

\section{Figures}

\section{Figure 1}

A

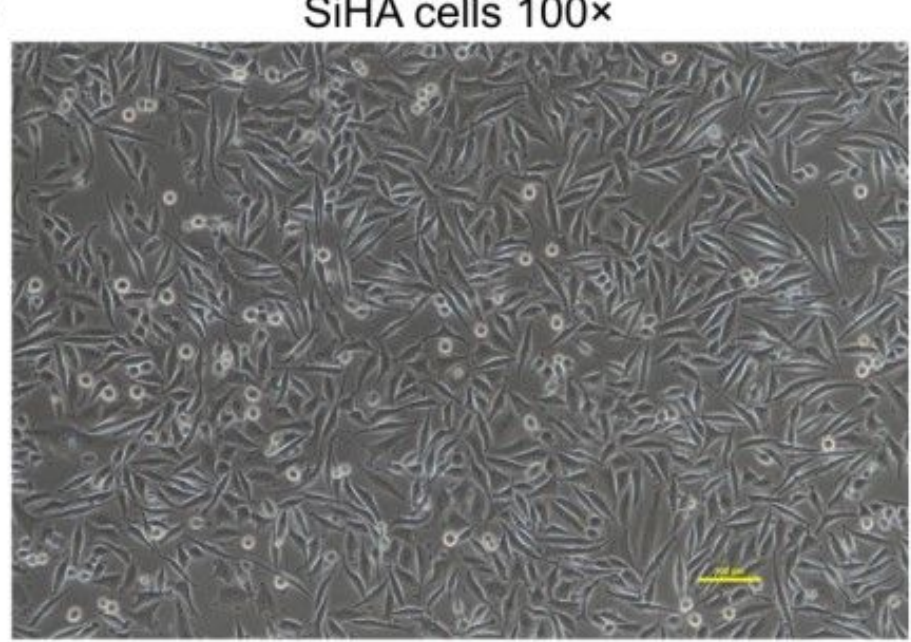

B

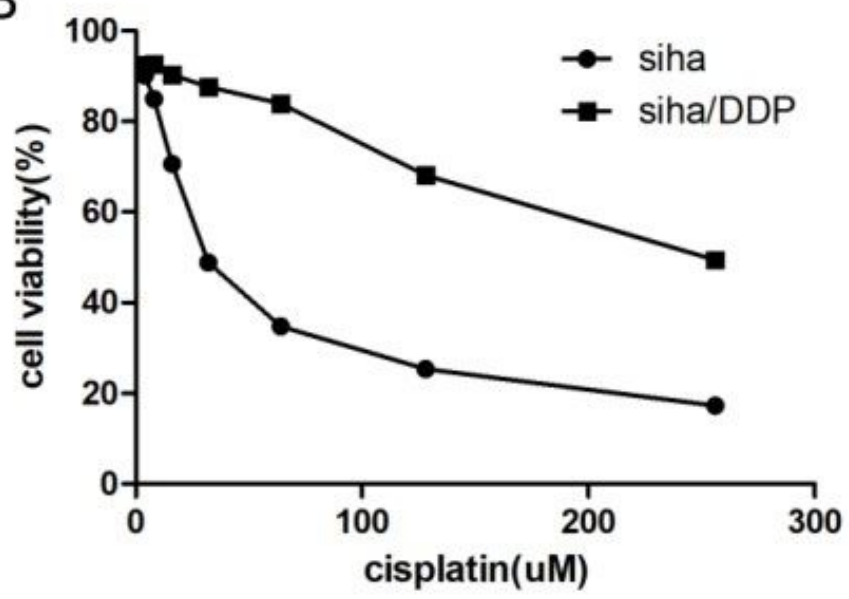

SiHA/DDP cells $100 \times$
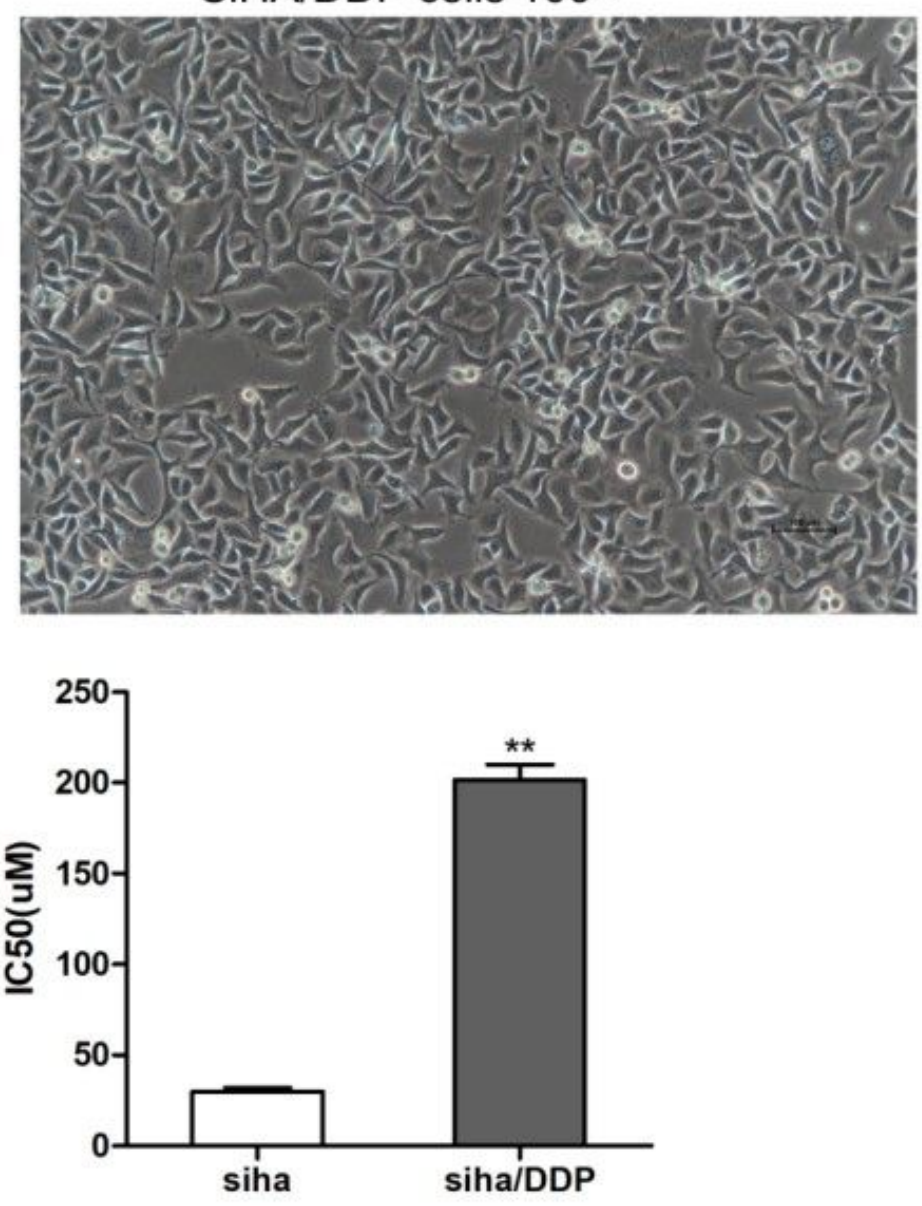


\section{Figure 1}

Morphological characteristics and drug sensitivity determination (A) The morphological difference between SiHA and SiHA/DDP cells. (B) CCK8 detects the cell viability of SiHA and SiHA/DDP cells under different cisplatin concentrations, and the IC50 of SiHA/DDP is significantly higher than that of SiHA cells.

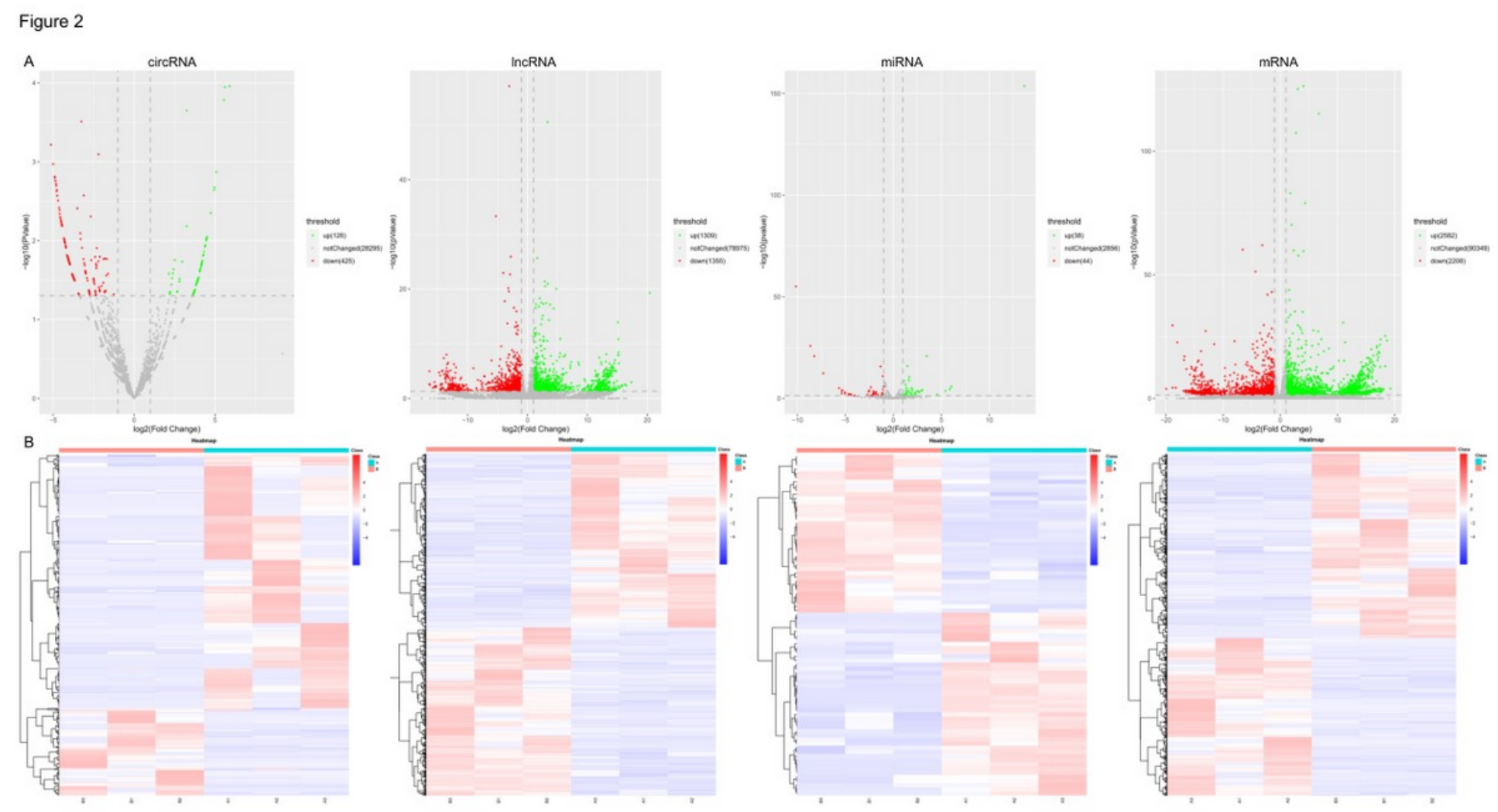

\section{Figure 2}

Expression profile of CircRNAs, IncRNAs, miRNAs and mRNAs Volcano map (A) and heat map (B) are used to show DE CircRNAs, IncRNAs, miRNAs and mRNAs in three DDP-sensitive and DDP-resistant CC cell lines. In the volcano map (A), the red, green, and gray dots represent the ncRNAs that are downregulated and up-regulated in SiHA/DDP and SiHA cells, respectively. x-axis: log2 ratio of ncRNA expression levels between SiHA/DDP and parental SiHA cells. Y-axis: ncRNA false discovery rate value (log10 conversion). Cluster analysis of ncRNA (B), red and blue expression: increase and decrease expression respectively. 
Figure 3
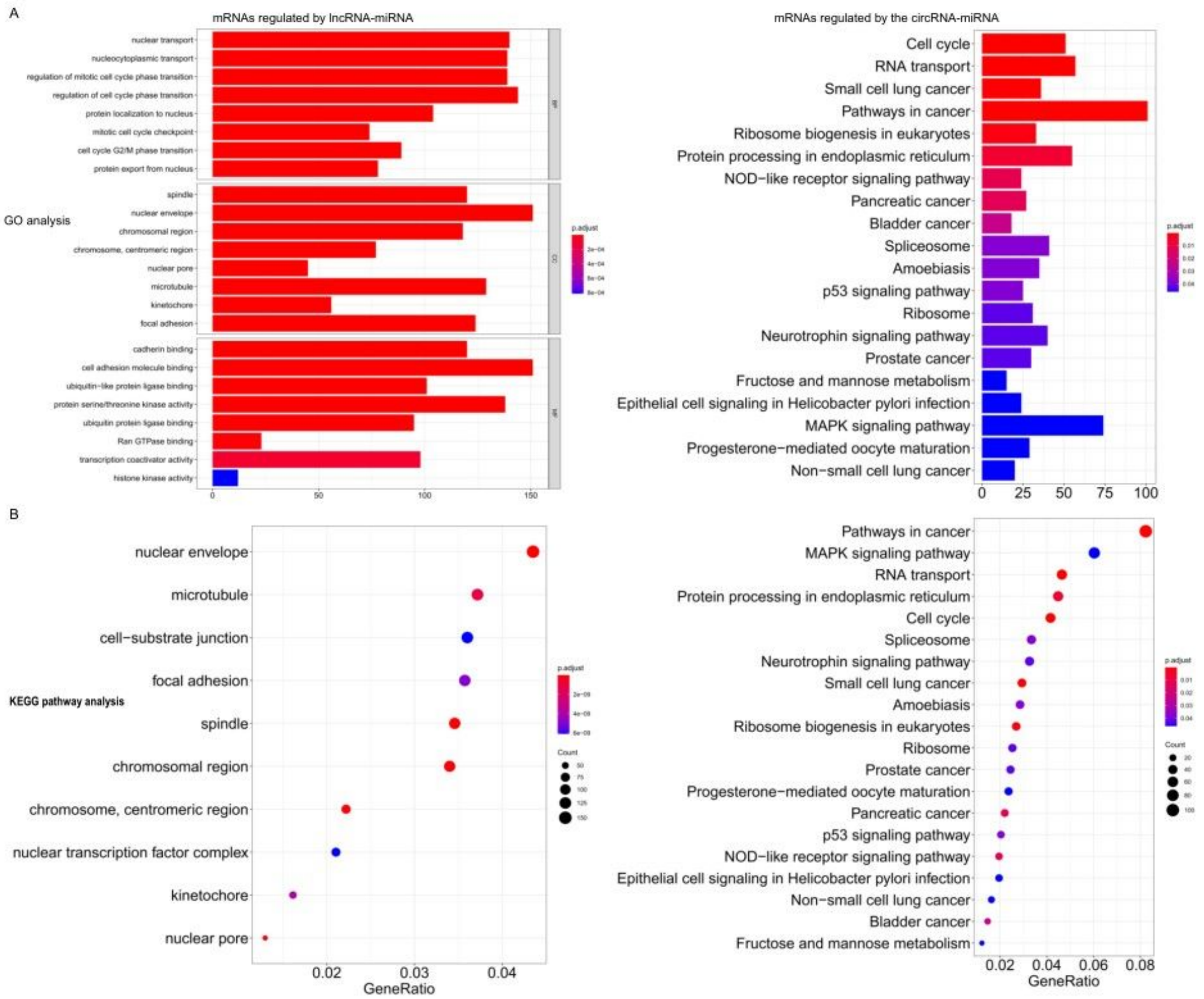

B

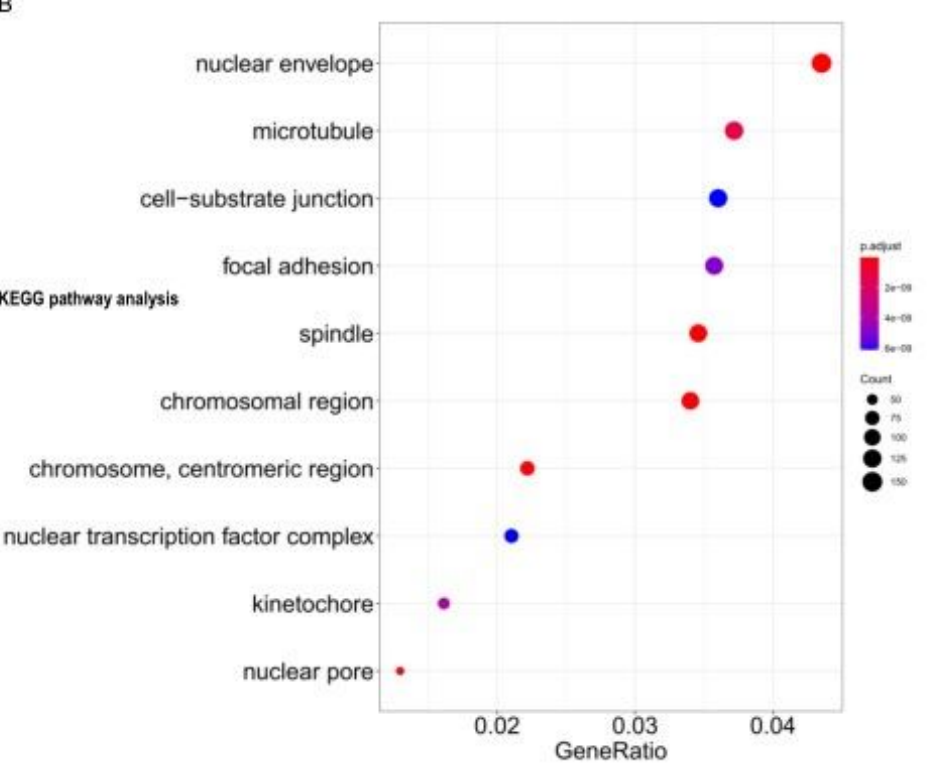

MAPK signaling pathway
RNA transport

ic reticulum

Cell cycle

ling pathway

ng cancer

eukaryotes

Ribosome

Prostate cancer

Pancreatic cance

53 signaling pathway

NOD-like receptor signaling pathway

Non-small cell lung cancer Bladder cancer

$\begin{array}{llll}0.02 & 0.04 & 0.06 & 0.08 \\ \text { GeneRatio } & 0.0\end{array}$

\section{Figure 3}

Bioinformatics analysis in the ceRNA network Analysis of GO and KEGG pathways of mRNA regulated by IncRNA-miRNA network (A) and circRNA-miRNA network (B). 
Figure 4

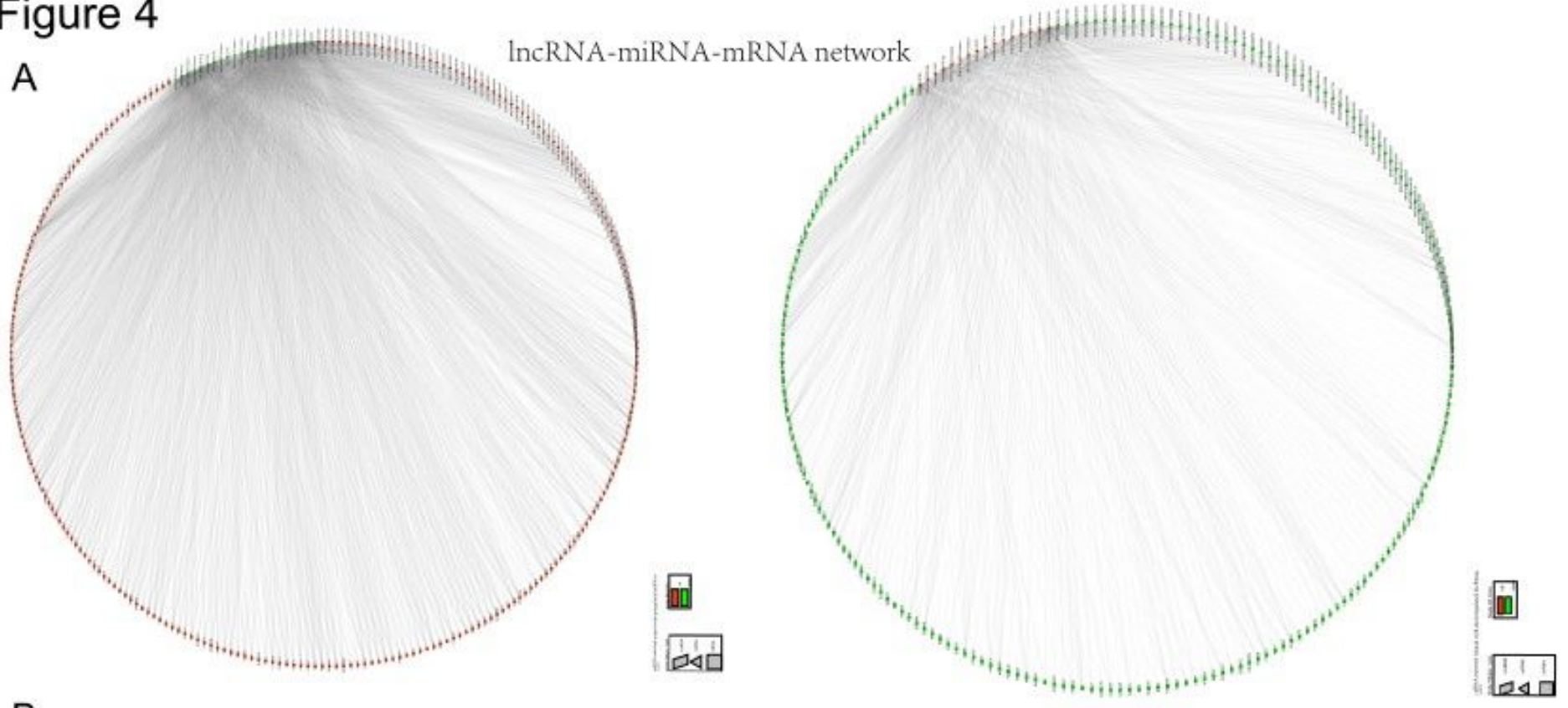

B

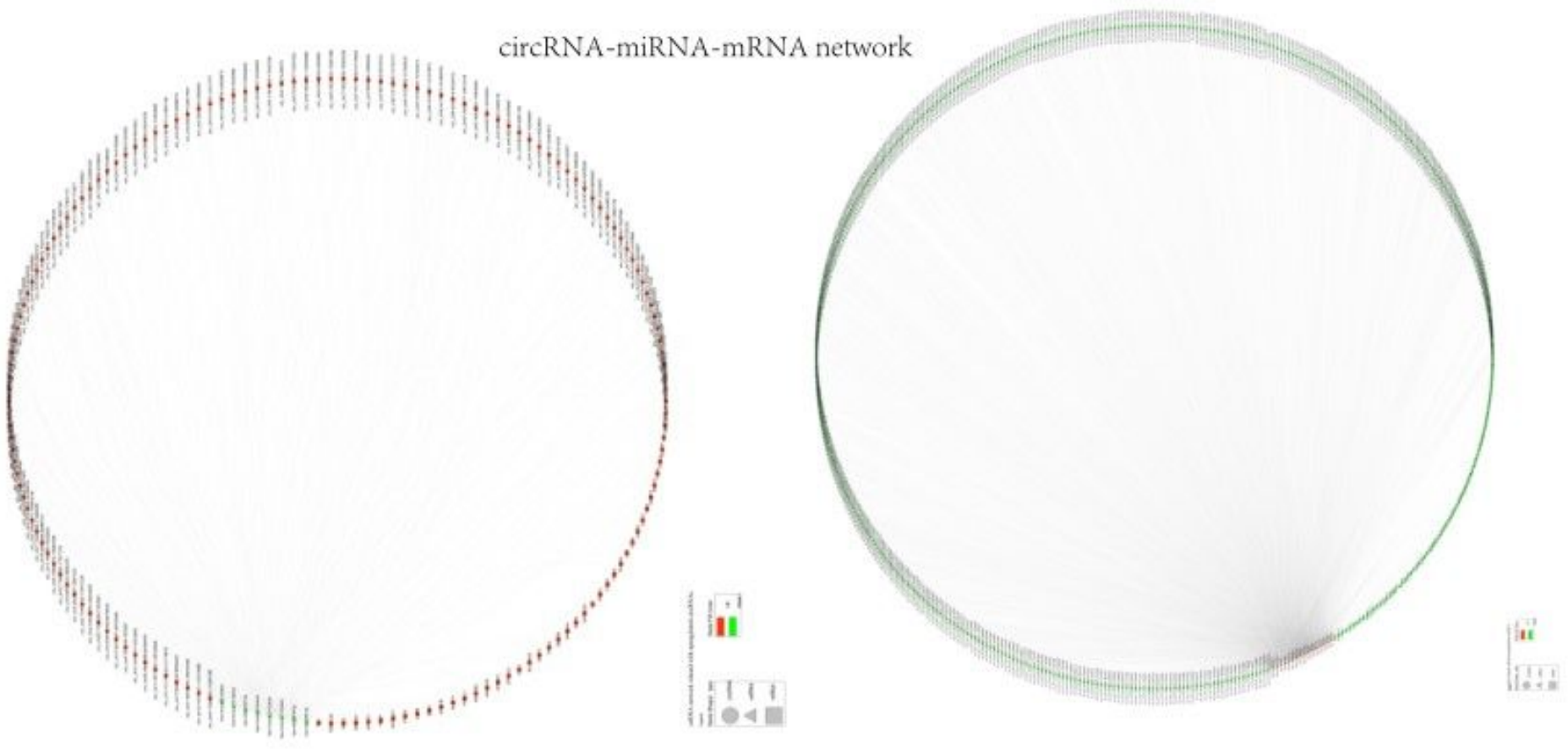

Figure 4

ceRNA network based on important genes related to drug resistance The IncRNA-miRNA-mRNA network (A) and circRNA-miRNA-mRNA network (B) are shown respectively. The diamonds represent IncRNAs, circular circRNAs, triangular miRNAs, and square mRNAs. Up-regulated red and bottom green, and the edges represent competitive interactions between them. 

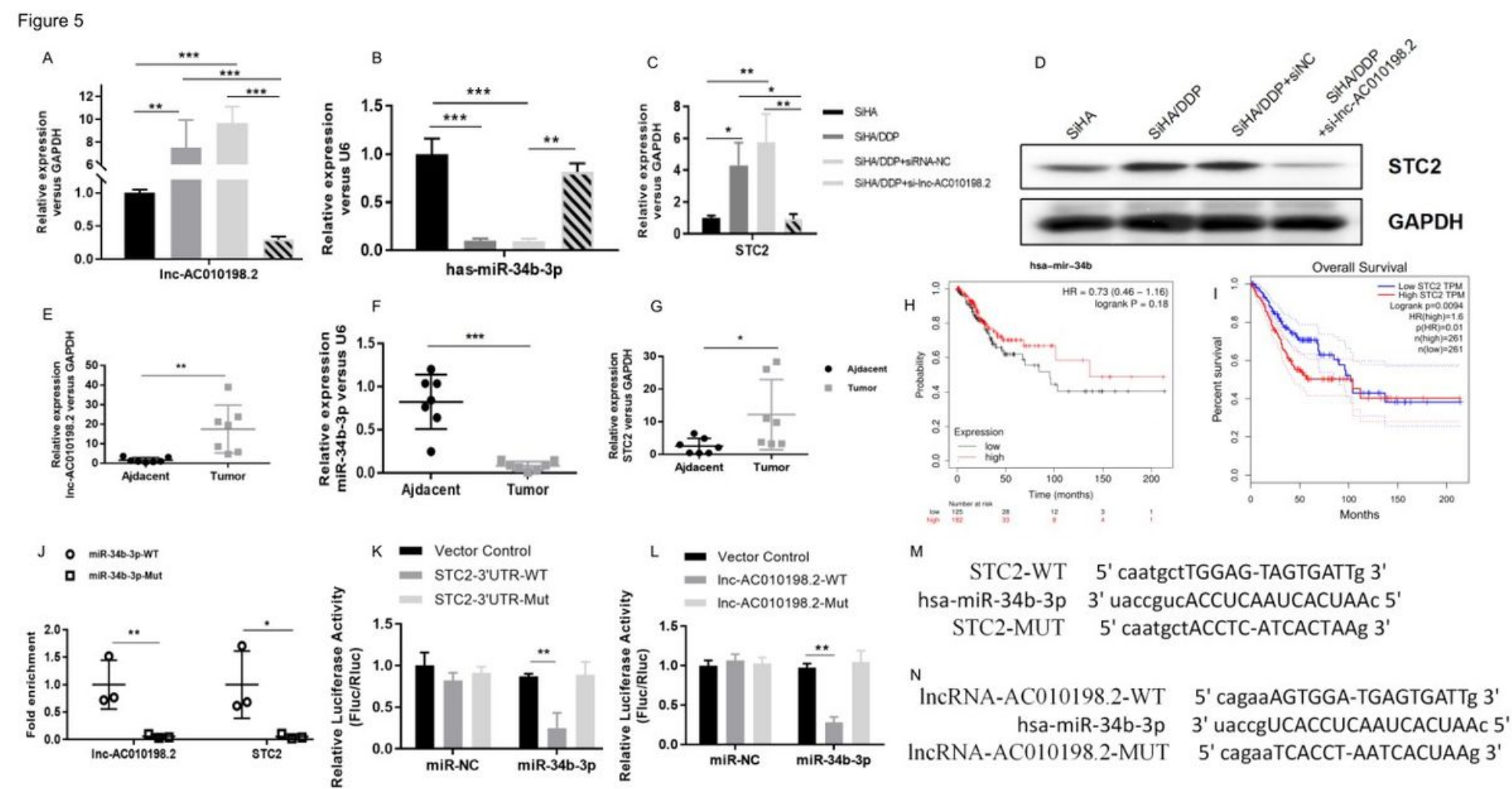

Figure 5

AC010198.2/hsa-miR-34b-3p/STC2 pathway was selected to verify the feasibility of the constructed IncRNA/miRNA/mRNA network (AG) The RNA expression levels of AC010198.2 (A and E), miR-34b-3p (B and F) and STC2 (C and G)-AC010198.2 and tissues in AC-treated CC cells were detected by real-time qPCR. (D) Compared with SiHA/DDP and SiHA/DDP-NC, the protein expression level of STC2 in CC cells treated with Si-AC010198.2 was checked by Western Blot. $(\mathrm{H}-1)$ High expression of miR-34b-3p indicates longer survival in the CESC of TCGA, and the opposite association with STC2 was observed. (J) RT-qPCR after RNA pull-down assay showed that compared with the control (NC-bio), the use of biotin-labeled miR34b-3p (miR-34b-3p-bio) probe can increase AC010198.2 and STC2 RNA expression) or miR-34b-3p-MutBio probe. (K) Luciferase activity analysis shows that the 3'UTR of STC2 can bind to miR-34b-3p. (M) Potential binding sites of STC2 and miR-34b-3p's 3'UTR. (L) The luciferase activity assay shows that miR34b-3p can bind to AC010198.2. (N) Potential binding sites of miR-34b-3p and AC010198.2. 
Figure 6

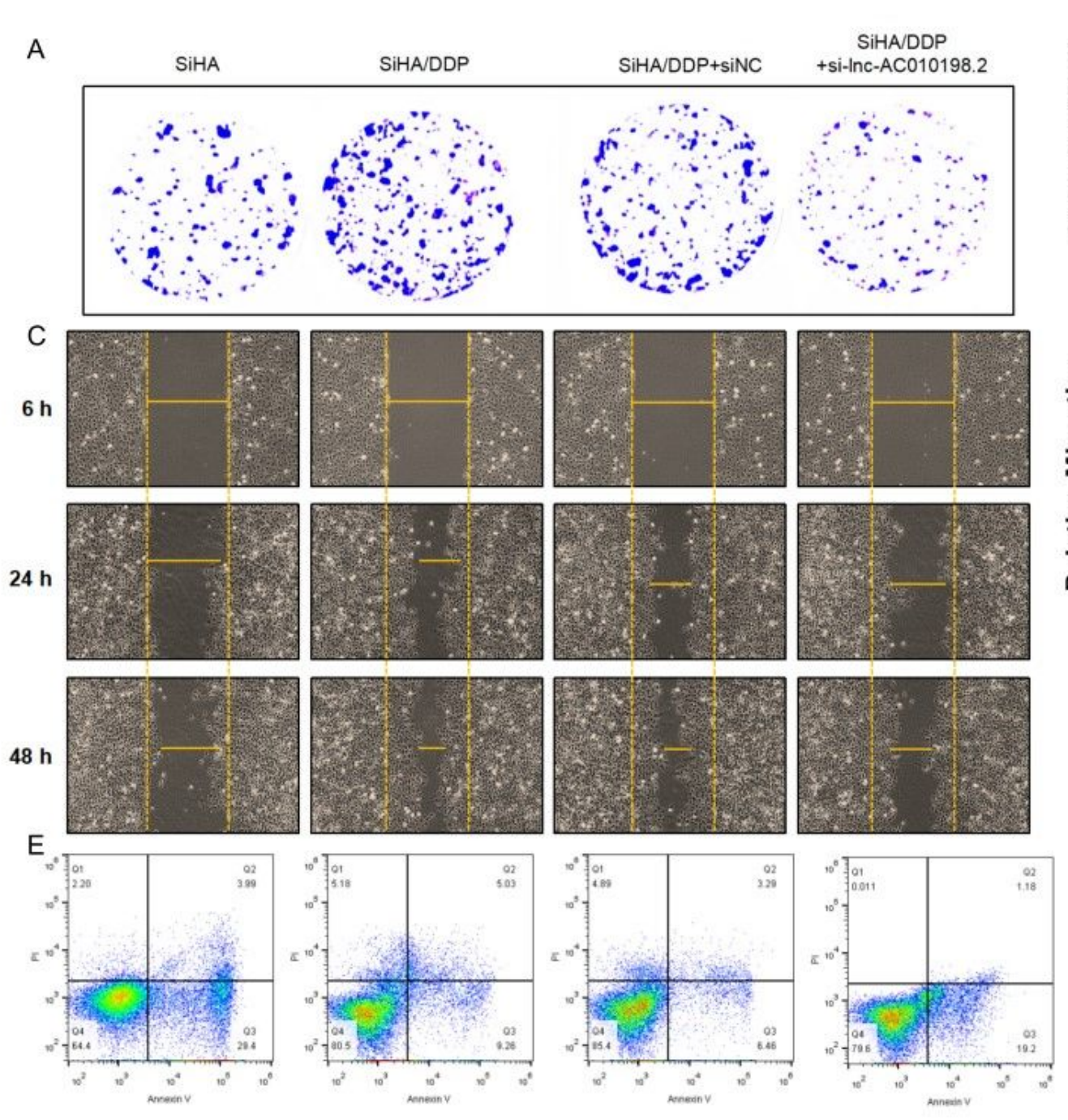

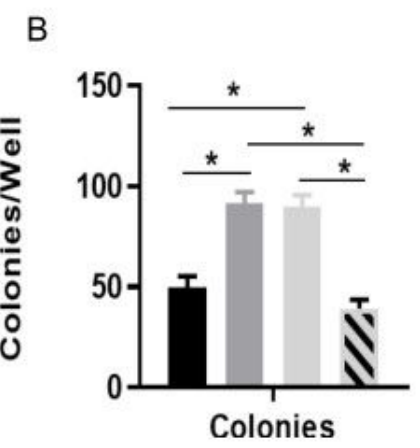

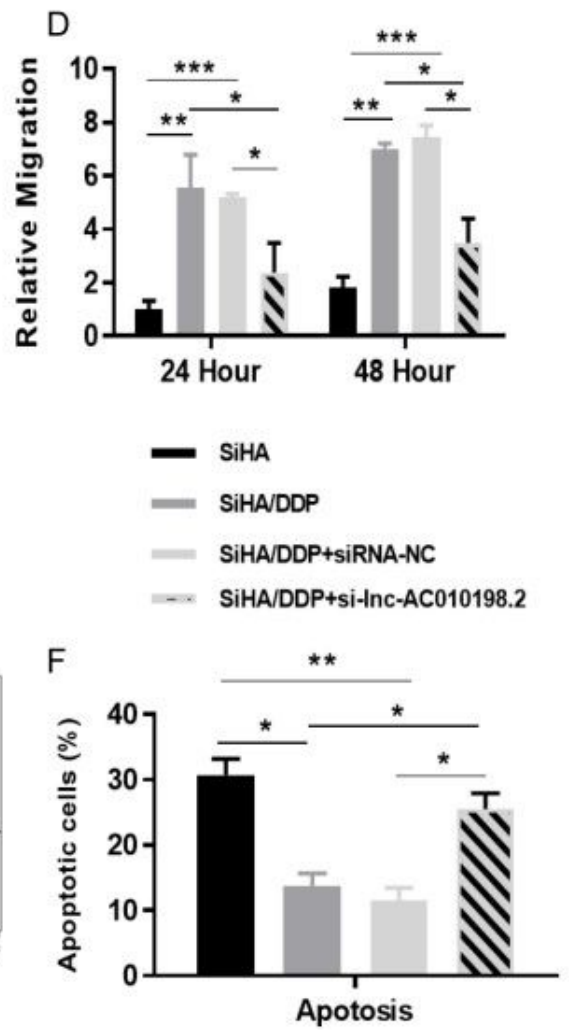

Figure 6

The function of AC010198.2 in CC cells after transfection with Si-AC010198.2 (A-B) The colony formation of CC cells is 7 days later. (C-D) Invasion efficiency of CC cells. (E-F) The apoptotic status of CC cells. ${ }^{*} p<0.05,{ }^{*} p<0.01,{ }^{* *} p<0.001$; SiHA/DDP or SiHA/DDP-NC with SiHA; SiHA/DDP-Si-AC010198.2 and $\mathrm{SiHA} / \mathrm{DDP}$ or SiHA/DDP-NC. The data are the mean \pm SD.

\section{Supplementary Files}

This is a list of supplementary files associated with this preprint. Click to download.

- TablesS.docx 